\title{
Extracción de perlilla (Symphoricarpos microphyllus H.B.K.) en el
} Nevado de Toluca

\section{Extraction of perlilla sticks (Symphoricarpos microphyllus H.B.K.) from the Nevado de Toluca Park}

\author{
Nancy Diana Anastacio Martínez', Esteban Valtierra Pacheco², Gabino Nava Bernal' y Sergio Franco Maass’,*
}

' Instituto de Ciencias Agropecuarias y Rurales. Universidad Autónoma del Estado de México.

2 Programa de Posgrado en Desarrollo Rural, Colegio *Autor de correspondencia. checolain@hotmail.com de Postgraduados.

\section{RESUMEN}

La presente investigación describe la organización social en la extracción y comercialización de la vara de perlilla (Symphoricarpos microphyllus H.B.K.) en tres comunidades del Área de Protección de Flora y Fauna Nevado de Toluca. La investigación se realizó a través de la observación participante, entrevistas semiestructuradas, transectos y muestreos aleatorios en áreas de extracción para determinar la densidad y características de las plantas. Los resultados muestran que el agente social detonador de la demanda del recurso es el Gobierno del Distrito Federal que requiere la vara de perlilla para la elaboración de escobas de vara rústicas destinadas a la limpieza pública. La organización social en torno a la extracción del recurso está dominada por intermediarios ajenos a las comunidades y, por lo tanto, son quienes obtienen el mayor beneficio económico de la extracción de perlilla. Ellos toman ventaja de las relaciones de amistad y parentesco con el acopiador y los líderes de los cortadores. Las entrevistas semiestructuradas con informantes clave permitieron identificar que la extracción de perlilla se ha intensificado presentando, en algunas zonas, problemas para la regeneración de la especie como resultado de la sobreexplotación y la aplicación de técnicas de corte inadecuadas. Esta situación podría ocasionar problemas graves de tipo ecológico, económico y social en esta área natural protegida.

PAlabras Clave: actores sociales, área natural protegida, escobas de vara, Gobierno del Distrito Federal, organización social.

\section{ABSTRACT}

This research describes the social process of extraction and commercialization of the perlilla sticks (Symphoricarpos microphyllus H.B.K.) in three communities of the Protected Wildlife Area of the Nevado of Toluca. This research was done through the participant observation, semi-structured interviews, transects and sampling of areas of extraction. The results show that the social agent who generates the demand of this resource is the Government of the Federal District to make rustic brooms used for public cleaning. The social organization around resource extraction is dominated by outside brokers and therefore they obtain most of the economic benefits the perlilla's extraction. They take advantage of their family and friend relationships with the leaders of cutters. The field work that identifies that the extraction of perlilla has been intensified that some areas already show problems of regeneration of this species that are linked to inadequate cutting technics. This situation could be causing severe ecological, economic and social problems in this natural protected area.

KEY WORDS: stakeholders, natural protected area, broom sticks, Government of Federal District, social organization.

\section{INTRODUCCIÓN}

La extracción, consumo y venta de Recursos Forestales No maderables (RFNM) en México contribuye de manera significativa a la economía de la población rural. Se estima que en el país existen cerca de 1000 diferentes RFNM que son extraídos para autoabasto o para su comercialización en mercados locales y regionales (FAO, 2004) (Marshall et al., 2006). De acuerdo con la FAO (1995a), el crecimiento 
de la población mexicana y su concentración en zonas urbanas y la revalorización de los RFNM han incrementado la demanda e intensificado su extracción, afectando la regeneración de las especies en su distribución natural. Entre los recursos no maderables que se extraen en el país destacan, por su relevancia económica, hongos y plantas comestibles, plantas medicinales, leña utilizada como combustible y otros productos comerciales como las resinas y el chicle (FAO, 1995b).

En 1936 se decretó el Parque Nacional Nevado de Toluca y, pese a gozar del máximo nivel de protección asignado por la legislación mexicana, esta área natural protegida ha sufrido un serio deterioro. En 2013, a iniciativa del Gobierno del Estado de México (GEM), se cambió la categoría con la finalidad de promover la recuperación y conservación de los recursos forestales, posibilitando la realización de actividades económicas que mejoren las condiciones de vida de la población local (Semarnat, 2013).

La perlilla (Symphoricarpos microphyllus H.B.K.) es un recurso forestal no maderable que se distribuye en los bosques de alta montaña, desde Nuevo México hasta Guatemala, incluyendo el Eje Neovolcánico Transversal de México (Calderón y Rzedowski, 2001). Suele crecer en bosque de oyamel (Abies religiosa (Kunth) Schltdl. et Cham.) a altitudes que oscilan entre $2700 \mathrm{~m}$ y $3500 \mathrm{~m}$ snm, sobre suelos de tipo andosol con alto contenido de materia orgánica y humedad (Benítez,1986).

En el presente siglo, la extracción de perlilla se ha convertido en una de las principales fuentes de ingresos para algunas comunidades del Área de Protección de Flora y Fauna Nevado de Toluca (APFFNT), debido a la demanda que genera la Zona Metropolitana de la Ciudad de México y, particularmente, el Gobierno del Distrito Federal (GDF), tanto para el mantenimiento de sus barredoras mecánicas como para el suministro de escobas a los trabajadores del servicio de limpia y para la fabricación de artesanías (Monroy et al., 2007). Dicha extracción es promovida por actores sociales externos a las comunidades, lo cual denota una desarticulación entre el objetivo de las políticas públicas en áreas protegidas que buscan impul- sar el desarrollo y calidad de vida de las comunidades rurales, la satisfacción de las verdaderas necesidades de la gente y la recuperación y conservación ambiental (Villalobos, 2000).

La demanda creciente de perlilla ha provocado una extracción intensiva del recurso en temporada de fructificación, afectando su regeneración, porque impide su correcto crecimiento y limita su propagación. Además, la extracción contraviene los preceptos de conservación y la normatividad para los cuales fue decretada el área de protección. La extracción de perlilla está ocasionando una perturbación de los ecosistemas y conflictos sociales entre los propietarios y usuarios de los terrenos de bienes comunales. En México, el estudio de los RFNM se ha centrado principalmente en aspectos biológicos de las especies, dejando de lado las cuestiones sociales inherentes a su extracción y comercialización. Es importante reconocer el papel de las comunidades y la forma en que los habitantes utilizan los recursos para obtener beneficios económicos (Monroy et al., 2007). Esto resulta especialmente relevante para el caso de la vara de perlilla, porque su extracción está afectando a un área natural protegida del Estado de México.

Existen diversos estudios relacionados con las plantaciones de vara de perlilla. Mendoza et al. (2012) presentan la estimación en el crecimiento y la altura de corte de la vara de perlilla en respuesta a un fertilizante, en San José del Rincón, Estado de México. De la misma manera, Mendoza et al. (2011) registran la calidad de la planta en una plantación de vara de perlilla ante la radiación solar y la supervivencia a través de un vivero de la Universidad Autónoma de Chapingo; y Monroy et al. (2007) plantean el proceso de apropiación y uso de la vara en el Corredor Biológico Chichinautzin, así como la propagación vegetativa de la planta al ser tratada con enraizador.

Desde el punto de vista social, varios estudios (Rodríguez e Higinio, 2011; Obama, 2002; De la Peña e Illsey, 2001) hacen referencia a la importancia que tienen los RFNM como sustento económico, alimenticio y cultural de muchas de las comunidades rurales. La organización social en torno a la extracción de los RFNM y las relaciones 
existentes entre los actores sociales tanto internos como externos quedan retratados en los estudios como el presentado por Sánchez y Valtierra (2003) donde analizan el entorno económico en el aprovechamiento de la palma camedor (chamaedorea spp.) en la Selva Lacandona en Chiapas y la problemática entre los grupos indígenas en torno a este proceso. Montañez et al. (2011) presentan el caso del aprovechamiento tradicional de una especie protegida (Litsea glaucescens Kunth) en Sierra del Laurel, Aguascalientes, donde se muestran los conflictos originados entre los comuneros y los empresarios por la obtención del recurso y, dada la temporalidad e importancia de la especie para el Domingo de Ramos, su extracción se vuelve intensiva en un corto tiempo lo que la ha llevado a estar en peligro de extinción.

\section{Objetivo}

El objetivo del presente estudio fue caracterizar la organización social en el proceso de extracción y comercialización de la vara de perlilla en tres comunidades del Área de Protección de Flora y Fauna Nevado de Toluca, en el Estado de México.

\section{MATERIALES Y MÉTODOS}

\section{Área de Estudio}

En el APFF Nevado de Toluca se destacan tres localidades en la extracción de vara de perlilla: La Peñuela y San José Contadero, ubicadas en el área de amortiguamiento y Buenavista localizada dentro de la zona núcleo (Conanp, 2012) (Fig. 1). En los tres casos, la población local complementa sus actividades económicas con la extracción de leña muerta y verde, hongos comestibles, plantas medicinales, plantas silvestres, frutos silvestres y algunos arbustos como la perlilla y la vara de cuete (Endara et al., 2012).

La Peñuela se ubica a una altitud promedio de 3040 $\mathrm{m}$ snm y cuenta con 655 habitantes (Inegi, 2010). Su principal actividad económica es la agricultura de riego, aunque existen predios agrícolas de temporal, predominando los cultivos de papa, avena y hortalizas. Buenavista se localiza a una altitud media de $3100 \mathrm{~m}$ snm y cuenta con

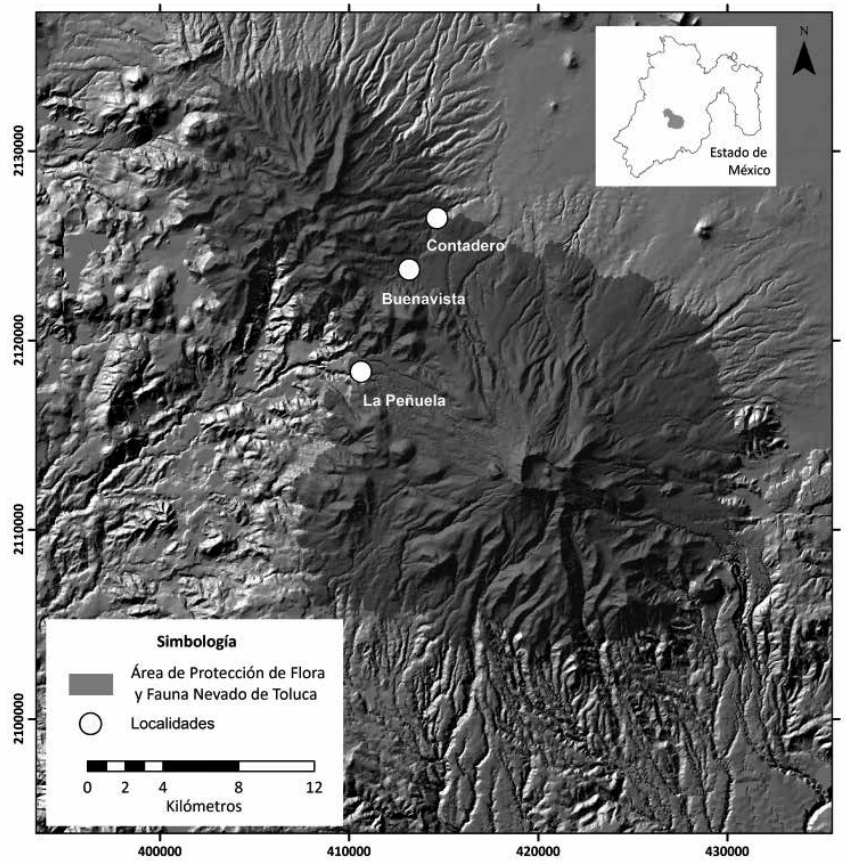

Figura 1. Ubicación de las comunidades de estudio.

Fuente: elaboración propia

560 habitantes (Inegi, 2010). La principal actividad económica es el pastoreo, aunque es posible encontrar pequeñas porciones de tierra cultivadas con papa y avena. Sus habitantes también tienen alternativas de trabajo fuera de la localidad, empleándose en la albañilería, aserraderos o como peones en temporada de cosecha. San José Contadero se ubica en la ladera norte del Volcán a una altitud promedio de $3040 \mathrm{~m}$ snm. Las principales actividades económicas son el cultivo de papa y la extracción de los recursos no maderables. El aprovechamiento de la leña y la extracción ilegal de madera para su comercialización son actividades comunes entre la población, debido a que la venta de estos se realiza tanto al interior como al exterior de la localidad (Conanp, 2012).

\section{Método}

El trabajo de campo se realizó de enero a octubre de 2013, tiempo durante el cual fue posible observar las tres etapas fenológicas de la perlilla en concordancia con el calendario propuesto por Monroy et al. (2007). La perlilla es una 
planta perenne cuyos frutos maduran y producen semilla entre marzo y junio; la floración se presenta de julio a septiembre y la fructificación va de octubre a febrero. Las técnicas de investigación empleadas fueron: la observación participante, entrevistas semiestructuradas, transectos y muestreo de sitios de extracción para determinar la densidad y características de la planta. La observación participante permitió identificar las técnicas de extracción, la cuantificación de varas y la calidad de las mismas. Se participó en todo el proceso incluyendo el corte, la elaboración de rollos, el cargado del camión y la comercialización.

Las entrevistas semiestructuradas se aplicaron a 12 personas involucradas en la extracción de perlilla de las tres localidades. Las preguntas estaban estructuradas con base en 4 ejes fundamentales: características del informante; conocimiento sobre la extracción de perlilla; funcionamiento de la organización social y participación en la distribución y comercialización.

Se realizarón tres transectos por localidad (Cámara y Díaz, 2013) con el fin de conocer las rutas de extracción y las zonas que, de acuerdo con los cortadores, presentan la mayor abundancia y calidad de perlilla. Para la ubicación de los transectos se utilizó un Sistema de Posicionamiento Global (GPS) y para la ubicación de otras rutas que no fue posible recorrer se utilizaron imágenes Google Earth. Se hicieron 9 muestreos sobre las rutas de extracción ( 3 por localidad). De manera aleatoria se estableció un cuadrante de $5 \mathrm{~m} \mathrm{x} 5 \mathrm{~m}\left(25 \mathrm{~m}^{2}\right)$ en una zona del transecto en que no se había realizado aún la extracción. Previo al corte de varas se realizó un conteó total obteniéndose: a) altura de los arbustos, b) número de varas por planta y c) el tipo de vara (retoño, joven o madura) con base en lo propuesto en MDSB (2006) para el muestreo de especies arbustivas. La abundancia se determinó por el conteo directo de inidviduos de perlilla encontrados en el área de muestreo expresándola en indivduos $/ \mathrm{m}^{2}$ (Vilchez, 2004). Una vez obtenida la información se permitió la entrada de los cortadores y se cuantificó el total de varas extraídas por arbusto. Los rollos obtenidos fueron pesados y medidos para determinar si cumplían con las características exigidas en la licitación (GDF, 2012).
Monroy et al. (2007) caracterizaron el calendario fenológico de la perlilla observándose en la figura 2 que el período de floración va de julio a septiembre, el de fructificación abarca cinco meses de octubre a febrero, finalmente, la semilla se produce y libera entre marzo y junio.

\section{RESULTADOS Y DISCUSIÓN}

\section{Aprovechamiento}

No todas las características establecidas en la NOM005-RECNAT-1997 (Semarnat, 2003: 31) son tomadas en cuenta por los cortadores. Si bien se presentan aprovechamientos menores a los establecidos en la normatividad (en promedio se aprovecha $46 \%$ en comparación con el $60 \%$ permitido), el corte se realiza durante la etapa de floración y semillación. De los muestreos realizados se contabilizó el número de arbustos, sus alturas, el total de varas por arbusto y el número y características de las varas aprovechadas (Tabla 1). La densidad promedio fue de 2.3 plantas $/ \mathrm{m}^{2}$. Los cortadores aprovechan las varas maduras y jóvenes omitiendo el corte de los retoños, lo que permite en cierta medida la regeneración de la especie.

\section{Periodo de extracción}

Los resultados de este trabajo demuestran que la extracción de perlilla en la región de estudio es intensiva y en distintos periodos en cada comunidad (Fig. 3). El periodo de corte en cada localidad depende de la percepción de los líderes de cuadrilla acerca de una mayor abundancia de la especie; la disponibilidad de cortadores y la decisión del

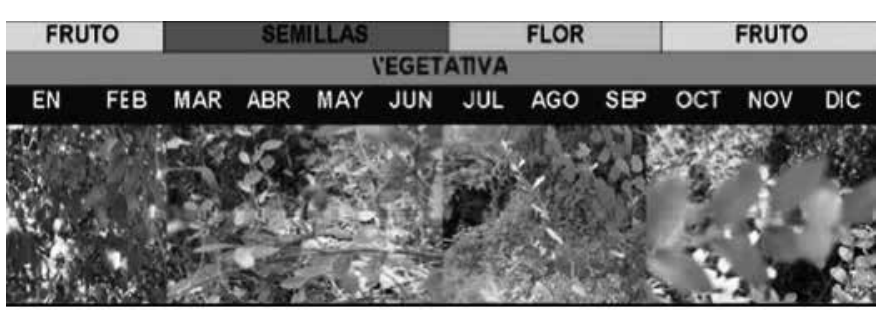

Figura 2. Calendario Fenológico de la perlilla (Symphoricarpos microphyllus H.B.K.).

Fuente: Monroy et al. (2007) 
acopiador local en función de la demanda. El desfase de corte entre las tres comunidades sucede porque entre los líderes de los cortadores existen relaciones de parentesco o amistad, lo cual les permite prestarse a los trabajadores. Considerando los periodos de las tres comunidades, la extracción prácticamente se extiende a todo el año.

La Secretaría de Medio Ambiente y Recursos Naturales (Semarnat), a través de la norma NOM-005-RECNAT-1997, señala en que etapa fenológica debe hacerse el aprovechamiento:

"Para promover el aprovechamiento (de tallos) de grupo de plantas en edad y tamaño homogéneo, solo se permitirá aprovechar como máximo el $60 \%$ de los tallos en madurez de cosecha. Cuando se presenten grupos en etapas de floración y semillación, sólo se deberá aprovechar el mismo porcentaje, con la finalidad de favorecer la reproducción por semilla”. (Semarnat, 2003: 31).

De acuerdo con lo encontrado por Monroy et al. (2007) (Fig. 2) y lo establecido por Semarnat, la época ideal para esta extracción es entre mayo y agosto. Sería ideal que los cortadores conocieran el calendario fenológico de la especie para cortar solo en las épocas adecuadas, permitiendo así la regeneración de la misma.

\section{Proceso de extracción}

El proceso de extracción-comercialización identificado en las tres áreas de estudio pertenecientes el APFFNT es el siguiente:

\section{a) Ubicación de los sitios de extracción}

La actividad se realiza tanto al interior como fuera de los límites del área natural protegida, principalmente sobre terrenos comunales. Esto implica que los grupos de cortadores deberían contar con el consentimiento de la comunidad y el permiso de la Semarnat. La gestión de estos permisos, sin embargo, solo se realiza en La Peñuela.

Los principales criterios que utilizan los cortadores para ubicar los parajes de extracción de perlilla son: la proximidad con caminos, veredas y parcelas de cultivo; la presencia de bosques abiertos de oyamel y barrancas con pendientes pronunciadas. Estos datos coinciden con lo publicado por Monroy et al. (2007) y Matesanz y Valladares (2009). Los autores afirman que la perlilla, siendo una especie ruderal, tiende a desarrollarse en hábitats alterados, aunque también es posible encontrarla en bosques de pino y encino. En la figura 4, se observa que los transectos seguidos por los cortadores corresponden a barrancas de fuerte pendiente cubiertas con bosque de oyamel y en proximidad a extensas zonas de cultivo.

La ubicación de las áreas de extracción variaba entre $5 \mathrm{~m}$ y $30 \mathrm{~m}$ de distancia a caminos y veredas. En las rutas de extracción de las localidades de Contadero y La Peñuela, la presencia del recurso era mayor a 5 metros de los caminos y tendía a disminuir hasta los $20 \mathrm{~m}$ o $30 \mathrm{~m}$. En el caso de Buenavista, la mayor cantidad de vara de perlilla estaba a 10 metros de la vereda y la menor cantidad a 30 metros.

\section{b) Selección de varas para la extracción}

De acuerdo con Hartmann y Kester (1985), la técnica correcta del corte de perlilla debe dejar las varas jóvenes y retoños para favorecer la regeneración de la planta. Pese a que los cortadores tienen conocimiento de esto, no siempre lo cumplen ya que deben sujetarse a las exigencias de los empresarios que, en ocasiones, demandan varas jóvenes porque se pueden almacenar por más tiempo.

En las licitaciones públicas que emiten las delegaciones del Gobierno del Distrito Federal se establecen las cantidades y la calidad de las varas, así como la forma de pago, pero no se especifica el estado de maduración de la vara.

Por ejemplo, en una licitación de 2012, el Comité Delegacional de Adquisiciones, Arrendamientos y Prestación de Servicios de la Delegación Coyoacán, establece:

"Adquisición de vara de perlilla de 1.20 a 1.50 mts. de altura en tercios y cada tercio se compone de 25 atados y cada atado se compone de 50 varas, para el periodo de enero a junio de 2012, para apoyar los trabajos de barrido manual tradicional en las calles que confinen esta delegación (Coyoacán). Monto a pagar \$5'000,000.00 (Cinco millones de pesos) por 7,194 tercios de perlilla. Coyoacán México a 13 de enero de 2012” (GDF, 2012) 
Las características que se consideran para el corte son: 1) el largo mínimo debe ser de $1.20 \mathrm{~m}$ (el largo máximo encontrado fue de $2.40 \mathrm{~m}$ ); 2) el diámetro del tallo o tronco principal de la mata debe ser mayor a $0.05 \mathrm{~m}$ (el grosor mínimo encontrado en campo fue de $0.07 \mathrm{~m}$ ); 3) la coloración debe ser marrón oscuro, lo que indica la edad de la vara (los cortadores de la zona no suelen extraer los retoños verdes, pero $43 \%$ de varas cortadas son rojizas o jóvenes y $57 \%$ son marrón oscuro o maduras) y; 4) la apariencia de lozanía sin rastros de deshidratación, descamación de su corteza, ni cortaduras a lo largo que puedan provocar el quiebre (los cortadores evitan el corte de este tipo de varas).

\section{c) Técnica de corte}

Durante los recorridos fue posible identificar dos técnicas de corte. Los cortadores de menor experiencia suelen cortar la planta de forma horizontal, mientras que los más experimentados aplican un corte diagonal a nivel del tronco principal. Esto último permite la regeneración de la especie y su propagación vegetativa, tal como lo señalan Mendoza, et al. (2012), Mendoza, et al. (2011), Monroy, et al. (2007) y Hartmann y Kester (1985). Es importante señalar que la cortadura diagonal estimula la produccion de raices pero, si no se realiza correctamente, se pone en riesgo la regeneración de la planta.

Los testimonios durante el trabajo de campo indican que en el año 2003, en las localidades de La Peñuela y Buenavista hubo una importante escasez de la especie que se prolongó por tres años. Los cortadores indicaron que esto se debió al aumento en la competencia entre grupos de cortadores y a la aplicación de técnicas inadecuadas de corte. Los arbustos eran cortados a lo ancho del tallo principal al nivel del suelo y de forma recta, impidiendo con ello la regeneración. Esta situación implicó que las personas dedicadas a la extracción emigraran a las ciudades de Toluca y México para emplearse como albañiles o permanecieran en sus comunidades empleándose como jornaleros.

\section{d) Formación de rollos para el secado}

Una vez cortadas las varas, se forman rollos que contienen entre 200 y 220 de ellas (Fig. 5). La variación en dicha cantidad depende de la calidad, es decir, cuando los rollos contienen 200 varas, existe una mayor proporción de material maduro y el tamaño, grosor, coloración y apariencia son los establecidos por el empresario. Si no se cuenta con la misma calidad, suelen intercalarse, ocultas en el centro del rollo, hasta 20 varas jóvenes, logrando con ello un grosor similar al de los rollos de óptima calidad. Cada cortador llegó a obtener entre 10 y 25 rollos de perlilla por día, dependiendo de su experiencia.

El armado de los rollos varía los fines de semana. Dado que se labora media jornada, en lugar de armar los rollos en el sitio de corte, se transportan las varas en camioneta a la casa del líder de cortadores y el resto de la tarde se arman los rollos.

\section{e) Almacenamiento de la vara de perlilla}

Una vez que los rollos han sido elaborados se ponen a secar al sol por un periodo de 15 días a 20 días (Fig. 6). Por las noches los rollos son cubiertos con plásticos o lonas para evitar que la vara pierda su flexibilidad y calidad a consecuencia de heladas y lluvias. En algunas ocasiones se construyen pequeños techados que permiten el paso de la luz solar y el viento sin que sea necesario cubrir los rollos todas las noches.

Si bien la perlilla se corta y almacena para satisfacer pedidos realizados con antelación, no existe garantía de que el intermediario recoja los rollos. Es importante señalar que una vez que la perlilla se ha secado, puede permanecer en almacenamiento por 3 semanas más aproximadamente, después de lo cual se echa a perder. Esto tiene una doble consecuencia negativa, afecta al cor-

\begin{tabular}{|c|c|c|c|c|c|c|c|c|c|c|c|}
\hline E & $\mathrm{F}$ & M & A & M & J & J & A & $\mathbf{s}$ & 0 & $\mathrm{~N}$ & D \\
\hline \multicolumn{12}{|c|}{ Buenavista } \\
\hline & & & & \multicolumn{3}{|c|}{ La Peñuela } & & & & & \\
\hline & & & & & & & \multicolumn{3}{|c|}{ Contadero } & & \\
\hline
\end{tabular}

Figura 3. Calendario de corte de perlilla en las comunidades de Buenavista, La Peñuela y Contadero.

Fuente: Trabajo de campo (2013). 
tador que no recibe el pago por su trabajo e incide en la abundancia de un recurso natural, que finalmente es desaprovechado.

La extracción de perlilla es una actividad económica secundaria que complementa el ingreso de algunas personas en las comunidades estudiadas. El proceso requiere de inversión de tiempo, esfuerzo físico para trasladarse a los sitios y para cargar los rollos y el riesgo por la exposición a mordeduras de serpiente y a ser arrestados por no contar con los permisos correspondientes de Semarnat.

\section{f) Comercialización de los rollos de perlilla}

El proceso de comercialización de la perlilla depende de la demanda del GDF que es el consumidor final. Las Delegaciones Políticas de GDF realizan licitaciones públicas para la adquisición de perlilla en las cuales pueden participar personas físicas y morales que sean capaces de suministrar la cantidad de rollos solicitados durante un período determinado. Una vez ganada la licitación, el intermediario firma un contrato donde se especifica la cantidad en tercios (cada tercio se compone de 25 atados de 50 varas cada uno), la calidad requerida de la vara y el monto en pesos que se compromete a pagar la Delegación por el recurso. El transporte del producto corre por cuenta del contratista o intermediario quien, de acuerdo con sus posibilidades, dispondrá de un camión propio o alquilado. Las comunidades generalmente surten quincenalmente entre 2500 y 4000 rollos de perlilla, que pesan entre 4 toneladas y 8 toneladas.

El contratista o intermediario no suscribe un contrato directamente con los cortadores, sino que realiza un pedido vía telefónica una semana antes de recoger el producto. En esa llamada se acuerdan los sitios de carga, las cantidades que cada comunidad surtirá al camión y el pre-

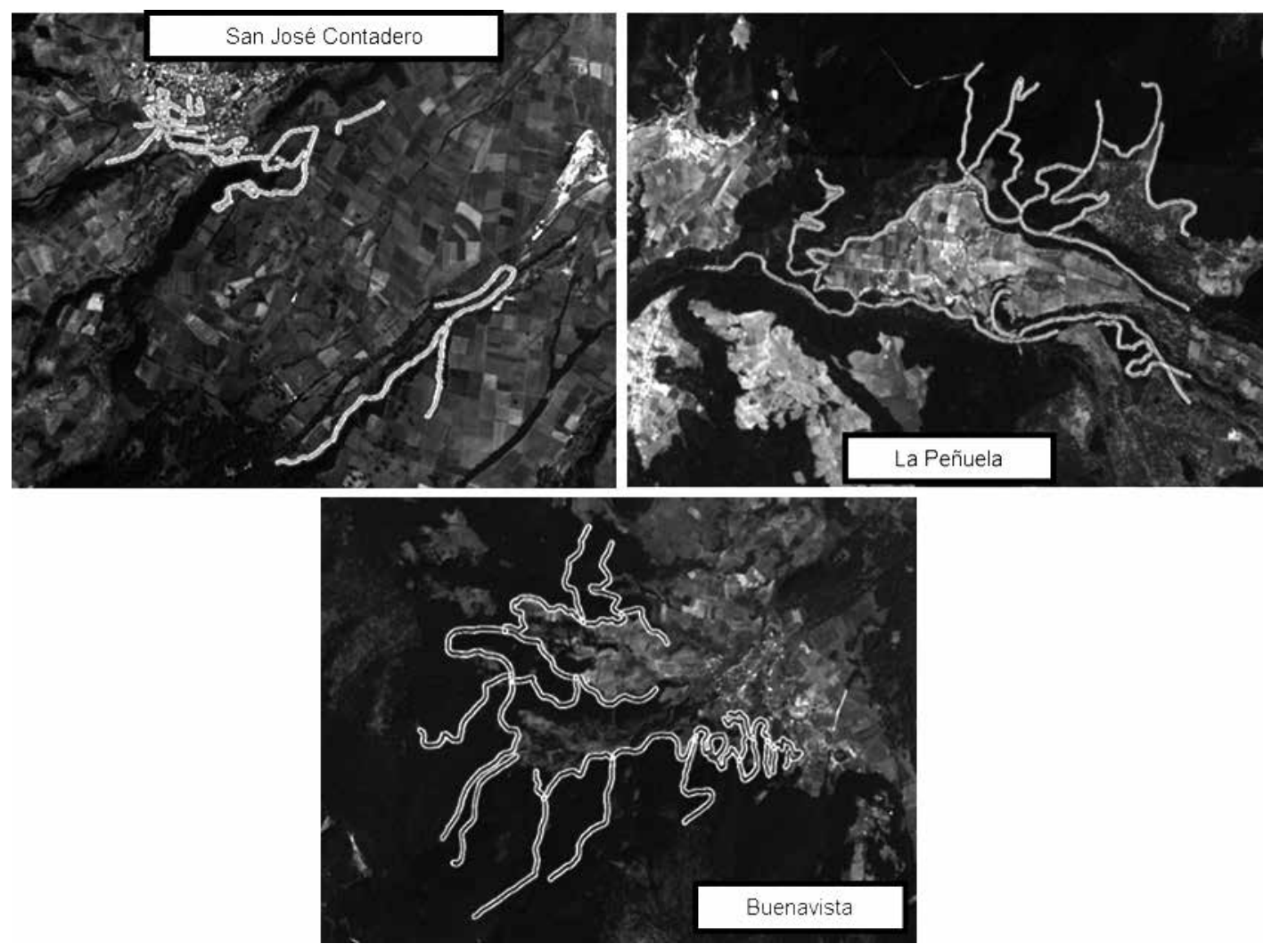

Figura 4. Rutas de extracción en las tres comunidades del APFFNT. 


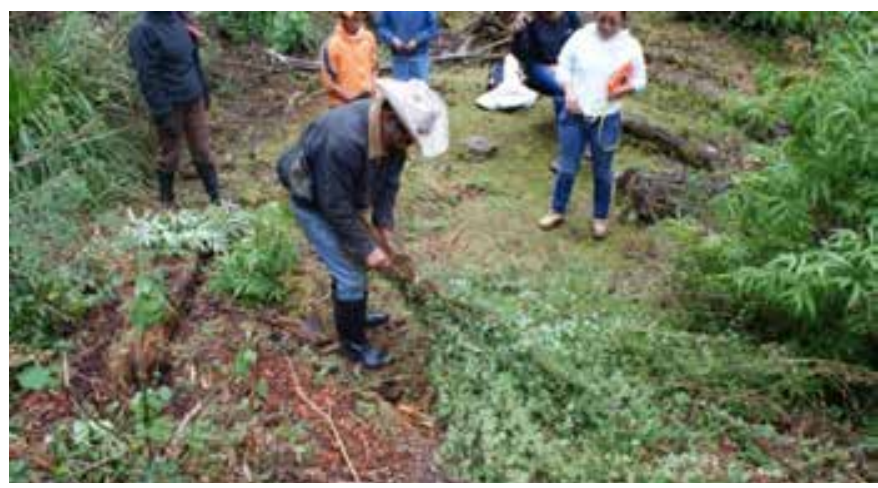

FIgURA 5. Formación de rollos de perlilla

Fuente: Trabajo de campo (2013).

Tabla 1. Aprovechamiento de vara de perlilla.

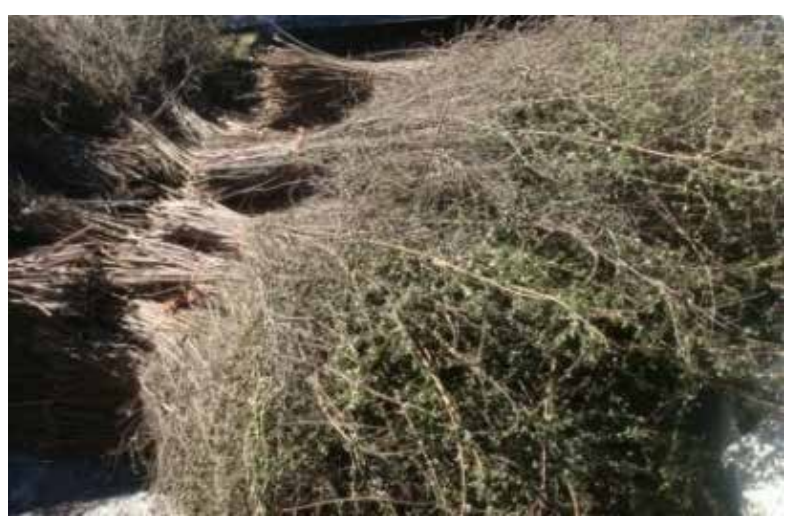

Figura 6. Exposición de la vara de perlilla a la luz solar. Fuente: Trabajo de campo (2013).

\begin{tabular}{ccccccc}
\hline Localidad & $\begin{array}{c}\text { No. De } \\
\text { arbustos }\end{array}$ & \multicolumn{2}{c}{ Altura promedio de arbusto } & \multicolumn{2}{c}{ Promedio de arbusto } \\
\hline & & Mínima & $\overline{\mathrm{x}}$ & Máxima & Total de varas & Varas cortadas \\
\cline { 3 - 7 } La Peñuela & 39 & $1.08 \mathrm{~m}$ & $1.76 \mathrm{~m}$ & $2.66 \mathrm{~m}$ & 27 & 15.7 \\
Buenavista & 54 & $1.02 \mathrm{~m}$ & $1.66 \mathrm{~m}$ & $2.67 \mathrm{~m}$ & 16 & 6.2 \\
San José & 80 & $0.76 \mathrm{~m}$ & $1.75 \mathrm{~m}$ & $2.29 \mathrm{~m}$ & 25 & 16.2 \\
Contadero & & & & & & \\
\hline
\end{tabular}

Fuente: Trabajo de campo (2013)

cio total por los rollos. Una vez que los rollos son entregados al camión, el transportista entrega el dinero al acopiador local quien procede al pago de los cortadores. Es evidente que, ante la falta de un contrato legal, tanto los líderes como los cortadores se encuentran indefensos ante cualquier incumplimiento del intermediario.

Durante el periodo de estudio en 2013, el precio por rollo de 200 varas de perlilla pagado a los cortadores variaba entre MXN\$10.00 y MXN\$15.00 (equivalente a USD\$ 0.82 y USD\$ 1.24 , a una tasa de cambio de MXN\$12.13 por USD\$1.00 al 15 de mayo de 2013, que es el punto medio del periodo de investigación). Esta variación dependía de la cantidad de rollos y la calidad de los mismos. Un cortador inexperto que produce un mínimo de 10 rollos de baja calidad (con más varas jóvenes que maduras) obtenía un pago diario de MXN\$100.00 pesos. Esto representaba el total de los ingresos en temporada de corte para la mayoría de los cortadores, debido a que cuando terminaba el corte de perlilla en esa localidad, buscaban trabajo como jornaleros para la cosecha de papa, maíz y otros cultivos.

Cuando los cortadores guardaban alguna relación de parentesco con el acopiador local podían recibir hasta MXN\$17.00 pesos por rollo, siempre y cuando cumplieran con la calidad necesaria del producto.

En la licitación del GDF para la adquisición de vara de perlilla para la Delegación Coyoacán emitida en 2012 (GDF, 2012), se estipulaba un pago de 5 millones de pesos por 7194 tercios. En este contexto el monto recibido por el empresario por un tercio era de MXN\$695.00, lo que 
representaba un ingreso de MXN\$111.20 pesos por rollo. Si se toma en cuenta que los costos promedio de transportación ascendían a MXN\$0.64 pesos y que el empresario pagaba MXN\$23.00 pesos al acopiador local, su ingreso neto estimado por rollo era de MXN\$87.56 pesos, es decir, $78.7 \%$ del total. El acopiador local pagaba al líder de los cortadores MXN\$17.00 pesos por rollo lo que le dejaba un remanente de MXN\$6.00 pesos $(5.4 \%$ del valor total del rollo). El líder de los cortadores pagaba MXN\$10.00 por rollo, teniendo un remanente de MXN\$7.00 $(6.3 \%$ del valor total del rollo). El pago a los cortadores representaba $8.9 \%$ del pago total. Es evidente que no existen diferencias significativas en el porcentaje de ingresos que perciben los diferentes actores sociales de las comunidades, las variaciones se dan en términos de los volúmenes que se manejan en cada nivel.

Lo anterior es ejemplo de lo que Rello (2008) señala en relación con la pobreza del sector agrícola mexicano como consecuencia del desajuste de políticas públicas que no cubren las necesidades del campesino, lo que ha conllevado a las personas a emplearse en trabajos informales fuera de su lugar de origen o bien a explotar los recursos naturales con los que cuenta su comunidad. La falta de apoyo económico por parte del sector gubernamental ha incidido en que sean los actores sociales externos quienes inviertan en el aprovechamiento de los recursos naturales de las comunidades rurales (Torres, 2010; Merino, 2004).

El empresario realiza la entrega a las delegaciones del GDF llevando una hoja de registro donde se establece el total de rollos entregados, la cantidad que se pagó y la fecha. Al final de la temporada se hace un balance de lo que se ha pagado y de la cantidad que se ha surtido. Definitivamente, es el intermediario quien se queda con la mayor ganancia por la extracción del recurso.

\section{Implicaciones en relación con el aprovechamiento de la perlilla}

En la comunidad de Contadero, la especie se desarrolla en terrenos ejidales, comunales y propiedad privada; sin embargo, la toma de decisiones para la extracción de los recursos depende exclusivamente de los ejidatarios. En la asamblea del ejido se analiza la solicitud del intermediario que desea cortar la perlilla y por medio de votación deciden si se otorga el permiso correspondiente. En caso afirmativo, se fija la cantidad a pagar por el permiso de extracción que, en 2013 fue de aproximadamente $\$ 8,000.00$ pesos. Una vez concedido el visto bueno al interesado se extiende el acta de conformidad que ampara la extracción por un periodo de un año. Esta situación deja en claro que la perlilla se considera como un recurso común, casi de libre acceso. Su extracción desmedida puede conducir a procesos de deterioro de los bosques que Hardin (1968) señala como la Tragedia de los Comunes.

En la investigación se corroboró que los cortadores conocen muy bien el proceso para la extracción legal de la perlilla. En primera instancia deben gestionar la aprobación de la comunidad y de los posesionarios de los predios y posteriormente solicitar el permiso ante la Semarnat, para lo cual es necesario que presenten la manifestación de impacto ambiental en su modalidad específica. Si se cumplen todos los requisitos, la Semarnat emite el permiso, estableciendo el periodo y la cantidad de recursos que es posible extraer. Estos trámites son complicados y representan costos que los actores sociales no están dispuestos a asumir, por lo que evitan, hasta donde es posible, hacer trámites en las dependencias gubernamentales y optan por la vía de la ilegalidad.

Durante la entrevista, el líder de los cortadores admitió que tenía que cubrir un pago al comisariado ejidal cuando eran delatados por extraer el recurso en zonas de propiedad privada. Dicho pago dependía de la cantidad de rollos extraídos y oscilaba entre MXN\$200.00 y MXN\$800.00 pesos. En pláticas ocasionales con las personas de las comunidades, mostraron desinterés en la extracción de la perlilla porque es una actividad poco lucrativa (Merino, 2004). En este contexto, son personas ajenas a la localidad quienes promueven la extracción y, bajo la lógica de la máxima utilidad, se favorece la sobreexplotación del recurso.

Las políticas públicas en materia ambiental dentro de un área natural protegida son el instrumento bajo el cual se busca dar solución a los problemas sociales mediante 
mecanismos de gestión estatal y participativa, este último aspecto es al que se le da la menor consideración en el desarrollo y gestión de programas debido a la inexistencia de una articulación entre dichos programas y las necesidades reales de la gente (Herrera et al., 2015).

El ejemplo de dicha desarticulación son los apoyos dirigidos al sector agrícola dentro del área natural y la existencia de una licitación para el aprovechamiento de perlilla que no permite la competitividad económica de los actores locales por: 1) la falta de una organización social interna que les permita aprovechar y gestionar sus propios recursos (Figueroa, 2005); 2) la falta de información sobre el proceso de gestión de los permisos y el desconocimiento de personas clave que coadyuven en el proceso y; 3) una competencia en condiciones imperfectas por considerarse un sector social marginal (Figueroa, 2005). Es así como los actores sociales externos (empresarios) son quienes aprovechan dichas licitaciones como parte de una complicidad de actores gubernamentales para permitir la extracción de recursos en zonas rurales (Merino, 2004).

La información generada sobre el aprovechamiento de la vara de perlilla puede servir como base para el desarrollo de políticas públicas para su aprovechamiento en condiciones de garantizar su conservación al tiempo que se logran mayores beneficios para los actores locales (Rodríguez e Higinio, 2011).

\section{La reproducción de la perlilla}

Ante la necesidad de satisfacer la demanda de perlilla y evitar los problemas de deterioro ambiental de las áreas naturales se ha planteado la posibilidad de las plantaciones. Algunos pobladores locales consideran que esto permitiría garantizar su disponibilidad, minimizar los conflictos con los posesionarios de los predios y reducir los riesgos de la recolección como las mordeduras de serpientes. Un cortador de San José Contadero destinó un pequeño predio para la plantación de perlilla mediante estacas obtenidas directamente en las zonas tradicionales de corte (Quintero et al., 2008) y sin utilizar productos químicos o enraizadores, como lo descrito por Monroy et al. (2007), Mendoza et al.(2012) y Mendoza et al. (2011).
Se ha logrado que las varas jóvenes y maduras produzcan sus propias raíces mediante inmersión en agua durante 4 semanas. Esta experiencia le animó a continuar con plantaciones a pequeña escala y no descarta la idea de adquirir un terreno más amplio que le permita producir su propia perlilla. Dado lo incipiente de la iniciativa, sin embargo, se desconoce su viabilidad en términos económicos.

\section{CONCLUSIONES}

El Gobierno del Distrito Federal, a través de sus Delegaciones Políticas, es el detonador de la explotación de la perlilla en la APFFNT, porque la utiliza para la elaboración de escobas rústicas destinadas a limpieza de los espacios públicos.

La extracción de perlilla es una de las principales actividades económicas para las familias que se dedican al corte, porque representa en algunos casos el total de sus ingresos económicos durante la temporada. Esto provoca competencia entre cortadores y grupos de extracción. Sin embargo, esta actividad no tiene importancia para la mayoría de los ejidatarios que son los posesionarios del recurso, porque no es redituable y requiere inversión de tiempo.

Los cortadores no consideran para la extracción el calendario fenológico de la especie y la NOM-005-RECNAT-1997, esta se realiza casi todo el año. La mejor época de corte es cuando los tallos están maduros, las plantas ya han liberado la semilla e incluso cuando se presenta la floración. Entonces el período ideal sería entre los meses de mayo y agosto. Este desfase entre el calendario fenológico y el periodo de extracción puede impactar en la regeneración de la especie.

La técnica empleada para el corte de perlilla determina la posibilidad de regeneración de la especie. Los grupos de cortadores han aprendido que el corte diagonal es el adecuado para promover el brote de nuevas ramas, sin embargo, en el pasado la planta se cortaba horizontalmente en la base del tallo principal, lo que provocaba escasez del recurso, como lo mencionado por Sánchez y Valtierra (2003) en la extracción de la palma camedor. Con referencia a este mismo recurso se encuentra lo des- 
crito por Conabio (2006) donde presenta la condición física de los recursos naturales, que en algún momento fueron considerados como renovables, pero que a consecuencia de su excesiva extracción se han convertido en recursos en peligro de extinción o, en el peor de los casos, agotables.

La organización de los actores en torno a la extracción de la perlilla se basa en un sistema económico que explota las relaciones de amistad y parentesco que tienen los líderes de los grupos de cortadores en las comunidades de la APFFNT, lo que permite que la actividad del corte de perlilla se repita año tras año. Las comunidades rurales de forma interna no presentan una organización social que les permita por un lado acceder de forma legal a sus recursos y comercializarlos para elevar su calidad de vida y por el otro mantener el control sobre su manejo.

La desarticulación existente entre las políticas públicas y las necesidades de la gente como lo plantea Villalobos (2000), ha conducido a que los programas de desarrollo no beneficien directamente a las comunidades rurales, siendo los beneficiarios directos los empresarios en la extracción de los recursos naturales.

La existencia de un consentimiento para la extracción de vara de perlilla al interior del área natural protegida denota la contradicción entre los objetivos de las políticas públicas, por un lado se busca el desarrollo de las comunidades rurales y la calidad de vida de las personas a través del aprovechamiento de sus recursos, mientras que por el otro lado se busca la conservación de dichos recursos bajo un enfoque conservacionista.

Los intermediarios que venden al GDF son quienes se quedan con la mayor parte del beneficio económico de la extracción de perlilla. La derrama económica en la región es limitada y solo algunos de los pobladores de las comunidades obtienen un magro ingreso mientras dura la temporada de corte. Y aun dentro de estos, los parientes del líder reciben mejor pago que quienes no lo son. Los ejidos posesionarios de los predios donde se corta la perlilla, solo reciben un pequeño pago anual de los líderes de los grupos de cortadores por el permiso para extraer el recurso.
La generación de información básica sobre el uso y aprovechamiento de la vara de perlilla podría considerarse como un elemento en el desarrollo de policías públicas sobre el manejo de recursos forestales no maderables al interior del APFFNT considerando los conocimientos sobre la especie que poseen los cortadores.

\section{REFERENCIAS}

Benítez, G. 1986. Symphoricarpos microphyllus. Árboles y flores de Jalisco. México: Instituto de Ecología y Museo de Historia Natural de la Ciudad de México. 33 p.

Calderón, G. y J. Rzedowski. 2001. Symphoricarpos microphyllus. Flora Fanerogámica del Valle de México. Pátzcuaro, Michoacán. Conabio-INE. 739 p.

Cámara, R. y F. Díaz. 2013. Muestreo en transecto de formaciones vegetales de fanerófitos caméfitos (I): Fundamentos metodológicos. Estudios Geográficos 74(274):67-88.

Conanp (Comisión Nacional de Áreas Naturales Protegidas). 2012. Estudio previo justificativo para la modificación de la Declaratoria del Parque Nacional Nevado de Toluca, ubicada en el Estado de México. Comisión Nacional de Áreas Naturales Protegidas [En línea] http://www.toluca. gob.mx/sites/default/files/Nevado/Nevado\%20de\%20 Toluca\%202013.pdf [Último acceso: 6 febrero 2014].

Conabio. (Comisión Nacional para el Conocimiento y Uso de la Biodiversidad). 2006. Capital Natural y Bienestar Social. México. 71 p.

De la Peña. G. y C. Illsey. 2001. Los productos forestales no maderables: su potencial económico, social y de conservación. Ecológica 27:1-6.

Endara, A., G. Nava, S. Franco, A. Espinoza, B. Ordoñez y C. Mallén. 2012. Extracción de madera en el Parque Nacional Nevado de Toluca. Nota de investigación. Ciencias Forestales 3(11):81-90.

FAO (Food and Agriculture Organization of the United Nations). 1995a. Report of The International Experts Consultation on Non wood Forest Products. Non-Wood Forest Products 3. [En línea] http://www.fao.org/docrep/v7540e/ v7540e00.htm [Último acceso: 25 febrero 2014].

FAO (Organización de las Naciones Unidas para la Alimentación y la Agricultura). 1995b. Memoria - Consulta de 
expertos sobre productos forestales no madereros para América Latina y el Caribe. Dirección de Productos Forestales, FAO, Roma. Oficina regional de la FAO para América Latina y el Caribe. Serie Forestal No. 1. [En línea] http://www.fao.org/docrep/t2354s/t2354s00.htm [Último acceso: 25 febrero 2014].

FAO (Organización de las Naciones Unidas para la Alimentación y la Agricultura). 2004. Categorías de los productos forestales no maderables. [En línea] http://www.fao.org/ documents/es/detail/155970 [Último acceso: 25 febrero 2014].

Figueroa, V. 2005. América Latina: descomposición y persistencia de lo campesino, Problemas del desarrollo. Latinoamericana de Economía 36(142):27-50.

GDF (Gobierno del Distrito Federal). 2012. Adquisición de varada de perlilla. Comité Delegacional de Adquisiciones, Arrendamientos y Prestación de Servicios. Delegación Coyoacán. Coyoacán, México.

Hartmann, T. y D. Kester. 1985. Propagación de plantas. México: CECSA. 760 p.

Inegi (Instituto Nacional de Estadística Geografía e Informática). 2010. Censo de población y vivienda por localidad 2010. [En línea] http://www.inegi.org.mx/est/contenidos/ proyectos/ccpv/cpv2010/Default.aspx [Último acceso: 5 febrero 2014].

Marshall, E., K. Schreckenberg y C. Newton. 2006. Comercialización de productos forestales no maderables: Factores que influyen en el éxito. Conclusiones del Estudio de México y Bolivia e Implicancias Políticas para los Tomadores de Decisión. [En línea] http://www.odi.org.uk/sites/ odi.org.uk/files/odi-assets/publications-opinionfiles/3771.pdf [Último acceso: 20 febrero 2014].

Matesanz, S. y F. Valladares. 2009. Plantas ruderales. Ciencia y Sociedad 390:10-11

Mendoza B., F. García, D. Rodríguez y S. Castro. 2011. Radiación solar y calidad de planta en una plantación de vara de perlilla (Symphoricarpos microphyllus H.B.K.). Agrociencia 46(2):235-243.

Mendoza, C., M. López, D. Rodríguez, A. Velázquez y F. García. 2012. Crecimiento de la Vara de Perlilla (Symphori- carpos microphyllus H.B.K.) en respuesta a la fertilización y altura de corte. Agrociencia 46(7):719-729.

Merino, L. 2004. Conservación o deterioro. El impacto de las políticas públicas en las instituciones comunitarias y en los usos de los bosques en México. Semarnat, INECOL y CCMSS. México. 331 p.

MDSB (Ministerio de Desarrollo Sostenible). 2006. Norma técnica para aprovechamiento comercial sostenible de recursos forestales no maderables en bosques y tierras forestales naturales. República de Bolivia. Resolución ministerial (22):13-15.

Monroy R., G. Castillo y H. Colín. 2007. La perlita o perlilla Symphoricarpos microphyllus H.B.K. (Caprifoliceae) especie no maderable utilizada en una comunidad del Corredor Biológico Chichinautzin. Morelos, México. Polibotánica 23:23-36.

Montañez, M., E. Valtierra y S. Medina. 2011. Aprovechamiento tradicional de una especie protegida (Litsea glaucescens) en Sierra de Laurel, Aguascalientes, México. $\mathrm{Ra}$ Ximbai 7(2):155-172.

Obama, C. 2002. Estudio de productos forestales no maderables en tres mercados de Guinea Ecuatorial. Anales del Jardín Botánico de Madrid 59(2):275-285.

Quintero A., A. Rodríguez, E. Guizar y R. Bonilla B. 2008. Propagación vegetativa de la vara de perlilla (Symphoricarpos microphyllus H.B.K.) Ciencias Forestales y del Ambiente 4(1):21-26.

Rello, F. 2008. Inercia estructural y globalización: La agricultura y los campesinos, más allá del TLCAN. Grupo de trabajo sobre desarrollo y medio ambiente en las américas. Trabajo de Discusión No. 20. 36 p.

Rodríguez, J. y J. Higinio. 2011. Importancia de los productos forestales maderables y no maderables en los hogares de Puerto Nariño. Cuadernos de Desarrollo Rural 6(62):3152.

Sánchez, D. y E. Valtierra. 2003. La organización social para el aprovechamiento de la palma camedor (chamaedorea spp.) en la selva Lacandona, Chiapas. Agrociencia $37(5): 545-552$.

Semarnat. 2013. Decreto que reforma, deroga y adiciona diversas disposiciones del diverso publicado el 25 de enero de 
1936, por el que se declaró Parque Nacional la montaña denominada "Nevado de Toluca" que fue modificado por el diverso publicado el 19 de febrero de 1937 en el Diario Oficial de la Federación.1 de octubre de 2013. Secretaria de Medio Ambiente y Recursos Naturales. DCCXXI (1):47-62.

Semarnat (Secretaría de Medio Ambiente y Recursos Naturales). 2003. NOM-005-RECNAT-1997. Que establece los procedimientos, criterios y especificaciones para realizar el aprovechamiento, transporte y almacenamiento de corteza, tallos y plantas completas de vegetación forestal. Publicado originalmente el 12 de enero de 1995 y Reforma de nomenclatura Publicada en el Diario Oficial de la Federación. 23 de abril de 2003. CDXCVI (9):28-33.

Tapia, F., I. Vizcarra y F. Torres. 2015. Políticas públicas y actores locales: aportes para el estudio de la dimensión institucional de las Áreas Naturales Protegidas en México. In: G. Rivera H., A. Martínez C. y Y. Gheno H., coords. Ediciones EÓN, ICAR. Universidad Autónoma del Estado de México. México. p:167-188.
Torres, G. 2010. Intensidad de la pobreza alimentaria en las zonas rurales. Localización y nuevas perspectivas para el desarrollo rural. Estudios agrarios (44):47-61.

Vilchez, B. 2004. Muestreo de Zamia fairchildiana L D. Gómez: un producto maderable del bosque. Kurú: Revista Forestal 1(1):1-5.

Villalobos, I. 2000. Áreas naturales protegidas: instrumento estratégico para la conservación de la biodiversidad. Gaceta Ecológica 54:24-34.

Manuscrito recibido el 11 de julio de 2014.

Aceptado el 20 de abril de 2015.

Este documento se debe citar como:

Anastacio M., N.D., E. Valtierra P., G. Nava B. y S. Franco M. 2015. Extracción de perlilla (Symphoricarpos microphyllus H.B.K.) en el Nevado de Toluca. Madera y Bosques 21(2):103-115. 